\title{
On the topological stable rank of certain transformation group $C^{*}$-algebras
}

\author{
IAN F. PUTNAM† \\ Department of Mathematics, Statistics and Computing Science, Dalhousie University, \\ Halifax, Nova Scotia, Canada, B3H $3 J 5$
}

(Received 20 October 1987 and revised 14 February 1989)

\begin{abstract}
We consider the crossed product or transformation group $C^{*}$-algebras arising from actions of the group of integers on a totally disconnected compact metrizable space. Under a mild hypothesis, we give a necessary and sufficient dynamical condition for the invertibles in such a $C^{*}$-algebra to be dense. We also examine the property of residual finiteness for such $C^{*}$-algebras.
\end{abstract}

\section{Introduction}

Throughout this paper, we let $X$ denote a compact, totally disconnected metrizable space. Let $C(X)$ denote the $C^{*}$-algebra of continuous complex-valued functions on $X$. We say that a subset $E$ of $X$ is clopen if it is both closed and open, and we use $\chi_{E}$ to denote the characteristic function of $E$. We define a partition of $X$ to be a finite collection of pairwise disjoint clopen subsets whose union is all of $X$. Given a partition $\mathscr{P}$ of $X$, we let $C(\mathscr{P})$ denote the finite-dimensional $C^{*}$-subalgebra of $C(X)$ which is generated by $\left\{\chi_{E} \mid E \in \mathscr{P}\right\}$. Since the clopen sets generate the topology of $X, \cup C(\mathscr{P})$ is dense in $C(X)$, where the union is taken over all partitions $\mathscr{P}$.

We let $\phi$ be a homeomorphism of $X$. We obtain a *automorphism, also denoted by $\phi$, of $C(X)$ by defining $\phi(f)=f \circ \phi^{-1}$, for each $f$ in $C(X)$. We will consider the crossed product $C^{*}$-algebra $C(X) x_{\phi} \mathbb{Z}$ obtained from the action of the group of integers on $C(X)$ generated by $\phi$. (See 7.6.5 of Pedersen [4].) It is generated as a $C^{*}$-algebra by $C(X)$ and a unitary operator, which we denote by $u_{X, \phi}$ or by $u$ when no confusion will arise, satisfying $u f u^{*}=\phi(f)$, for all $f$ in $C(X)$.

We refer the reader to Blackadar [1] for standard facts regarding $K$-theory for $C^{*}$-algebras.

First of all, the $K$-theory of $C(X)$ is computable since it is an $A F C^{*}$-algebra (Effros [3] or Blackadar [1]). Specifically, $K_{1}(C(X))=0$ and $K_{0}(C(X))$ is isomorphic to $C(X, \mathbb{Z})$, the group of continuous $\mathbb{Z}$-valued functions on $X$, with positive cone the non-negative functions. Secondly, we may apply the Pimsner-Voiculescu six-term exact sequence [6] to completely determine the $K$-theory of $C(X) x_{\phi} \mathbb{Z}$ (also see Blackadar [1]). We summarize the results in the following theorem. 
THEOREM 1.1. (i) Let $i$ denote the inclusion of $C(X)$ in $C(X) x_{\phi} \mathbb{Z}$. Then $i_{*}: K_{0}(C(X)) \rightarrow K_{0}\left(C(X) x_{\phi} \mathbb{Z}\right)$ is surjective and its kernel is the image of id- $\phi_{*}$, considered as an endomorphism of $K_{0}(C(X))$.

(ii) If $X$ contains no nontrivial clopen $\phi$-invariant subsets, then $K_{1}\left(C(X) x_{\phi} \mathbb{Z}\right) \cong \mathbb{Z}$ and is generated by the class of $u$.

A subset of $X$ is said to be minimal for $\phi$ if it is a minimal non-empty, closed, $\phi$-invariant set. We let $S^{1}$ denote the circle and $M_{m}$ denote the $C^{*}$-algebra of $m$ by $m$ matrices.

In this paper, we wish to determine the topological stable rank of the $C^{*}$-algebra $C(X) x_{\phi} \mathbb{Z}$ (denoted tsr $\left(C(X) x_{\phi} \mathbb{Z}\right)$ ). We refer the reader to Rieffel [8] for a complete treatment of the topic, but we note that from 7.4 of $[8], \operatorname{tsr}\left(C(X) x_{\phi} \mathbb{Z}\right)$ is either 1 or 2. Also from [8], we see that our original problem reduces to the question of whether or not the invertible elements of $C(X) x_{\phi} \mathbb{Z}$ are dense.

We first consider the case when $X$ itself is minimal for $\phi$ in $\S 2$. We show that given $a_{1}, a_{2}, \ldots, a_{n}$ in $C(X) x_{\phi} \mathbb{Z}$ and $\varepsilon>0$, there is a unital $C^{*}$-subalgebra $A$ of $C(X) x_{\phi} \mathbb{Z}$ and $a_{1}^{\prime}, a_{2}^{\prime}, \ldots, a_{n}^{\prime}$ in $A$ such that $\left\|a_{i}-a_{i}^{\prime}\right\|<\varepsilon$, for $i=1, \ldots, n$, and $A$ is *-isomorphic to

$$
\left[C\left(S^{1}\right) \otimes M_{J_{1}}\right] \oplus M_{J_{2}} \oplus \cdots \oplus M_{J_{K}}
$$

for some integers $J_{1}, J_{2}, \ldots, J_{K}$.

This result is of interest in its own right (compare with the definition of $A F$ algebra), but as a corollary we show that the invertibles are dense in $C(X) x_{\phi} \mathbb{Z}$, when $X$ is minimal.

In $\S 3$, we turn to the general case. (Actually, we impose the very mild restriction that $X$ have no nontrivial clopen $\phi$-invariant subsets.) We obtain a necessary and sufficient condition on $(X, \phi)$ for the invertibles in $C(X) x_{\phi} \mathbb{Z}$ to be dense; namely, that there is exactly one minimal set for $\phi$. We also show that these conditions are actually equivalent to $C(X) x_{\phi} \mathbb{Z}$ having the cancellation property for finitely generated projective modules (see Rieffel [8]).

Finally, in $\S 4$, we examine the question of finiteness and residual finiteness of $C(X) x_{\phi} \mathbb{Z}$. Using the work of Pimsner [5], we produce a necessary and sufficient condition on $(X, \phi)$ for the $C^{*}$-algebra $C(X) x_{\phi} \mathbb{Z}$ to be residually finite. We also produce a somewhat surprising example of a residually finite $C(X) x_{\phi} \mathbb{Z}$ where the invertibles are not dense.

\section{The minimal case}

Here, we deal with the case when $X$ is minimal for $\phi$.

TheOREM 2.1. Suppose that $X$ is miminal for $\phi$. Given a partition $\mathscr{P}$ of $X$ and an $\varepsilon>0$, there is a unital $C^{*}$-subalgebra, $A \subseteq C(X) x_{\phi} \mathbb{Z}$, which is ${ }^{*}$-isomorphic to

$$
\left[C\left(S^{1}\right) \otimes M_{J_{1}}\right] \oplus M_{J_{2}} \oplus \cdots \oplus M_{J_{K}}
$$

(for some integers $\left.J_{1}, J_{2}, \ldots, J_{K}\right)$ and such that $C(\mathscr{P}) \subseteq A$ and there is a unitary $u^{\prime}$ in $A$ such that $\left\|u-u^{\prime}\right\|<\varepsilon$. 
The proof is lengthy, so we defer it for the moment while we point out the following consequences.

Corollary 2.2. Suppose that $X$ is minimal for $\phi$. Given $a_{1}, a_{2}, \ldots, a_{n} \in C(X) x_{\phi} \mathbb{Z}$, there is a unital $C^{*}$-subalgebra $A \subseteq C(X) x_{\phi} \mathbb{Z}$ of the same form as in 2.1 and $a_{1}^{\prime}$, $a_{2}^{\prime}, \ldots, a_{n}^{\prime} \in A$ such that $\left\|a_{i}-a_{i}^{\prime}\right\|<\varepsilon$, for all $i=1,2, \ldots, n$.

The proof of 2.2 is a trivial consequence of 2.1 .

CORollary 2.3. If $X$ is minimal for $\phi$, then the invertible elements of $C(X) x_{\phi} \mathbb{Z}$ are dense.

Proof. Given an element $a$ in $C(X) x_{\phi} \mathbb{Z}$ and $\varepsilon<0$, we find $A$ and $a^{\prime}$ as in 2.2, with $\left\|a-a^{\prime}\right\|<\varepsilon / 2$. From Proposition 1.7, Theorem 3.3 and Theorem 5.2 of Rieffel [8], we may find an invertible element, $a^{\prime \prime}$, of $A$ such that $\left\|a^{\prime \prime}-a^{\prime}\right\|<\varepsilon / 2$. Thus, $\left\|a-a^{\prime \prime}\right\|<$ $\varepsilon$ and $a^{\prime \prime}$ is invertible.

For a $C^{*}$-algebra $B$ and a positive integer $n$, we let $G L_{n}(B)$ denote the invertible elements of $M_{n} \otimes B$. Also, $G L_{n}^{0}(B)$ denotes the connected component of the identity in $G L_{n}(B)$.

COROLLARY 2.4. If $X$ is minimal for $\phi$, then for all positive integers $n$, the natural map

$$
G L_{n}\left(C(X) x_{\phi} \mathbb{Z}\right) / G L_{n}^{0}\left(C(X) x_{\phi} \mathbb{Z}\right) \rightarrow K_{1}\left(C(X) x_{\phi} \mathbb{Z}\right)
$$

is an isomorphism.

Proof. As we noted in Theorem $1.1, K_{1}\left(C(X) x_{\phi} \mathbb{Z}\right)$ is generated by the class of $u$. Therefore, the natural map above is surjective for all $n$.

We wish to show that the natural map

$$
G L_{n}(B) / G L_{n}^{0}(B) \rightarrow K_{1}(B)
$$

is injective, for $B=C(X) x_{\phi} \mathbb{Z}$. Let $a$ be an element of $G L_{n}\left(C(X) x_{\phi} \mathbb{Z}\right)$. It is well-known that the set of invertible elements in a Banach algebra is open. Together with Proposition 2.1, this implies that we may find a unital $C^{*}$-subalgebra $A \subset$ $C(X) x_{\phi} \mathbb{Z}$, as in 2.1, and an element $a^{\prime}$ in $G L_{n}(A)$ such that $a$ and $a^{\prime}$ are homotopic in $G L_{n}\left(C(X) x_{\phi} \mathbb{Z}\right)$. Thus, it suffices to consider the case $B=A$, with $A$ of the form in 2.1 .

Since $A$ is a direct sum of matrix algebras over $\mathbb{C}$ and $C\left(S^{1}\right)$, we see that it sufficient to consider the cases $B=\mathbb{C}$ and $B=C\left(S^{1}\right)$. For the former, $G L_{n}(\mathbb{C})=$ $G L_{n}^{0}(\mathbb{C})$ and so the result is trivial. As for $B=C\left(S^{1}\right)$, we may view elements of $G L_{n}\left(C\left(S^{1}\right)\right)$ as continuous functions from $S^{1}$ into $G L_{n}(\mathbb{C})$ and in this way identify $G L_{n}\left(C\left(S^{1}\right)\right) / G L_{n}^{0}\left(C\left(S^{1}\right)\right)$ with $\pi_{1}\left(G L_{n}(\mathbb{C})\right)$. In (10.17) on p. 204 of Whitehead [11], it is shown that $\pi_{1}\left(G L_{n}(C)\right) \simeq \mathbb{Z}$ and that the path $f(t)=\mathrm{e}^{2 \pi \mathrm{i} t} p+\left(I_{n}-p\right)$, for $t \in[0,1]$, where $p$ is any rank one projection in $M_{n}$, is a representative of a generator of the group. From this we conclude that the natural map is an isomorphism in the case $B=C\left(S^{1}\right)$.

Proof of Theorem 2.1. If $X$ is finite, then $C(X) x_{\phi} \mathbb{Z} \cong C\left(S^{1}\right) \otimes M_{J}$, where $J$ is the cardinality of $X$. So the result is trivial in this case. 
Let us consider the case when $X$ is infinite. Any finite orbit would be a closed invariant subset of $X$ and so we see that the action is free. Thus, $C(X) x_{\phi} \mathbb{Z}$ is simple. (See Theorem 5.15 of Zeller-Meier [12].) It will be useful to have a concrete representation of $C(X) x_{\phi} \mathbb{Z}$.

We fix a $\phi$-invariant probability measure on $X$ (such a measure always exists-see Theorem 1, p. 37 of Corfeld et al. [2]), and consider the Hilbert space $L^{2}(X)$, suppressing the measure in our notation. If $Y$ is a clopen subset of $X$, there is a natural decomposition $L^{2}(X)=L^{2}(Y) \oplus L^{2}(X-Y)$.

We define a covariant representation of our $C^{*}$-dynamical system as follows. Let $C(X)$ act on $L^{2}(X)$ by multiplication:

$$
(f \xi)(x)=f(x) \xi(x), \quad \text { for } f \in C(X), \xi \in L^{2}(X) \text { and } x \in X .
$$

We also define a unitary operator $u$ on $L^{2}(X)$ by

$$
(u \xi)(x)=\xi\left(\phi^{-1}(x)\right), \quad \text { for } \xi \in L^{2}(X) \text { and } x \in X .
$$

Then $C(X) x_{\phi} \mathbb{Z}$ is the $C^{*}$-algebra generated by $C(X)$ and $u$. (See 7.6.4 of Pedersen [4]. We use the fact that $C(X) x_{\phi} \mathbb{Z}$ is simple to conclude that this representation is faithful.)

We begin with the partition $\mathscr{P}$ and $\varepsilon>0$. Choose an integer $N>0$ such that $\pi / N<\varepsilon$

Choose a point $x_{0}$ in $X$ arbitrarily. The points $x_{0}, \phi\left(x_{0}\right), \ldots, \phi^{N}\left(x_{0}\right)$ are all distinct so we may choose a clopen neighbourhood $Y$ of $x_{0}$ satisfying

(i) $Y, \phi(Y), \ldots, \phi^{N}(Y)$ are pairwise disjoint, and

(ii) For each $n=0,1, \ldots, N$, the set $\phi^{n}(Y)$ is contained in an element of $\mathscr{P}$.

We will construct an approximation to $\phi$. This technique was first developed by Versik [9, 10] and may be viewed as an analogue of the Rokhlin Lemma [2] in a topological setting.

We define a function $\lambda: Y \rightarrow \mathbb{Z}$ by

$$
\lambda(y)=\inf \left\{n \geq 1 \mid \phi^{n}(y) \in Y\right\}, \quad \text { for } y \in Y .
$$

The minimality of $\phi$ implies that the positive itterates of any point are dense and since $Y$ is open, this is well-defined. It is straightforward to see that $\lambda$ is upper (lower) semi-continuous because $Y$ is open (closed). Thus, $\lambda$ is continuous. As $Y$ is compact, so is $\lambda(Y)$. Therefore, $\lambda(Y)$ is finite; say $\lambda(Y)=\left\{J_{1}, J_{2}, \ldots, J_{K}\right\}$. Notice that by (2.1), each $J_{k}$ is greater than or equal to $N$.

We define clopen sets $Y(k, j)$, for each $k=1,2, \ldots, K$, and each $j=1, \ldots, J_{k}$, by

$$
Y(k, j)=\phi^{j}\left(\lambda^{-1}\left(J_{k}\right)\right) \text {. }
$$

It is immediate from the definitions that we have

$$
\begin{gathered}
\phi(Y(k, j))=Y(k, j+1), \quad \text { for } 1 \leq j<J_{k}, \\
\bigcup_{k=1}^{K} Y\left(k, J_{k}\right)=Y, \quad \text { and } \\
\phi\left(\bigcup_{k=1}^{K} Y\left(k, J_{k}\right)\right)=\bigcup_{k=1}^{K} Y(k, 1)=\phi(Y) .
\end{gathered}
$$


It follows from (2.2) and (2.4) that the union of all $Y(k, J)$ is invariant under $\phi$. This union is also closed and therefore must be all of $X$. Thus, $\{Y(k, j) \mid 1 \leq k \leq K$, $\left.1 \leq j \leq J_{k}\right\}$ is a partition of $X$. It is clear that by dividing up the individual towers, $\left\{Y(k, j) \mid 1 \leq j \leq J_{k}\right\}$, we may make this partition finer than $\mathscr{P}$. (This will increase the value of $K$, but does not affect any of the properties (2.2)-(2.4)). We do this so that $C(\mathscr{P}) \subset C\left(\mathscr{P}_{0}\right)$, where

$$
\mathscr{P}_{0}=\left\{Y(k, j) \mid 1 \leq j \leq J_{k}, 1 \leq k \leq K\right\} .
$$

We now define a finite dimensional unital $C^{*}$-subalgebra $A_{0} \subset C(X) x_{\phi} \mathbb{Z}$. In fact, $A_{0}$ will be *-isomorphic to

$$
M_{J_{1}} \oplus M_{J_{2}} \oplus \cdots \oplus M_{J_{K}} .
$$

To do this, it suffices to define a system of matrix units by

$$
e_{i j}^{(k)}=\chi_{Y(k, i)} u^{i-j}, 1 \leq k \leq K, 1 \leq i, j \leq J_{k} .
$$

It is straightforward to verify that, for fixed $k,\left\{e_{i j}^{(k)} \mid 1 \leq i, j \leq J_{k}\right\}$ forms a complete system of matrix units for $M_{J_{k}}$ and that the projections

$$
p_{k}=\sum_{i=1}^{J_{k}} e_{i i}^{(k)}, \quad 1 \leq k \leq K,
$$

form a partition of unity in $C(X) x_{\phi} \mathbb{Z}$. Also notice that

$$
C\left(\mathscr{P}_{0}\right)=\operatorname{span}\left\{e_{i i}^{(k)} \mid 1 \leq k \leq K, 1 \leq i \leq J_{k}\right\},
$$

so that $C(\mathscr{P}) \subset A_{0}$. For any positive integer $n, M_{n}$ is generated (as a $C^{*}$-algebra) by the diagonal matrices and the single nilpotent matrix consisting of ones immediately below the diagonal and zeros elsewhere. In our case, this allows us to observe that $A_{0}$ is the $C^{*}$-subalgebra of $C(X) x_{\phi} \mathbb{Z}$ generated by $C\left(\mathscr{P}_{0}\right)$ and $u \chi_{X-Y}$.

Let us return to the dynamical situation and define homeomorphisms $\phi_{0}$ and $\psi_{0}$ of $X$ by

$$
\begin{gathered}
\psi_{0}(x)= \begin{cases}\phi(x) & \text { for } x \notin Y=\bigcup_{k} Y\left(k, J_{k}\right) \\
\phi^{1-J_{k}}(x) & \text { for } x \in Y\left(k, J_{k}\right)\end{cases} \\
\phi_{0}=\psi_{0}^{-1} \circ \phi .
\end{gathered}
$$

Observe that $\psi_{0}$ is the disjoint union of $K$ periodic homeomorphisms, and that $\phi_{0}$ is the identity off of $Y$. (Its restriction to $Y$ is the first return map of $\phi$ with respect to $Y$.)

We define unitary operators $u_{0}$ and $v_{0}$ on $L^{2}(X)$ by

$$
\begin{aligned}
& \left(u_{0} \xi\right)(x)=\xi\left(\phi_{0}^{-1}(x)\right), \\
& \left(v_{0} \xi\right)(x)=\xi\left(\psi_{0}^{-1}(x)\right),
\end{aligned}
$$

for $\xi \in L^{2}(X)$ and $x \in X$. It is easily checked that

$$
v_{0}=\sum_{k=1}^{K}\left[\sum_{i=2}^{J_{k}} e_{i, i-1}^{(k)}+e_{1, J_{k}}^{(k)}\right],
$$

so that $v_{0} \in A_{0}$, and that $v_{0} u_{0}=u$ so that $u_{0} \in C(X) x_{\phi} \mathbb{Z}$. Since $\psi_{0}=\phi$ off of $Y, v_{0}=u$ and $u_{0}=I$ on $L^{2}(X-Y)$. 
We now let $Z=Y\left(1, J_{1}\right)$ and repeat the procedure beginning by defining $\lambda^{\prime}: Z \rightarrow \mathbb{Z}$ and obtaining $K^{\prime}, J_{1}^{\prime}, \ldots, J_{K^{\prime}}^{\prime}$ and clopen $Z(k, j)$ which satisfy conditions analogous to (2.2)-(2.4). We also insist that the partition $\mathscr{P}_{1}=\left\{Z(k, j) \mid 1 \leq k \leq K^{\prime}, 1 \leq j \leq J_{k}^{\prime}\right\}$ is finer than $\mathscr{P}_{0}$ and that $C\left(\mathscr{P}_{1}\right)$ contains $\chi_{Y}$.

As before, we obtain a finite dimensional $C^{*}$-subalgebra, $A_{1} \subset C(X) x_{\phi} \mathbb{Z}$. (We will not need to write down matrix units for $A_{1}$.) As before for $A_{0}$, we may describe $A_{1}$ as the $C^{*}$-subalgebra generated by $C\left(\mathscr{P}_{1}\right)$ and $u \chi_{X-Z}$. Since $\mathscr{P}_{1} \supset \mathscr{P}_{0}$ and $Z \subset Y$, it is clear that $A_{0} \subset A_{1}$.

Define homeomorphisms, $\phi_{1}$ and $\psi_{1}$, of $X$ and unitaries, $u_{1}$ and $v_{1}$, on $L^{2}(X)$ in an analogous way so that $u=v_{1} u_{1}, v_{1} \in A_{1}, u_{1} \in C(X) x_{\phi} \mathbb{Z}$ and $v_{1}=u$ and $u_{1}=I$ on $L^{2}(X-Z)$.

The unitary operator $u_{1}$ is the identity except on

$$
L^{2}(Z)=L^{2}\left(Y\left(1, J_{1}\right)\right)=e_{J_{1}, J_{1}}^{(1)} L^{2}(X)
$$

so the $C^{*}$-algebra generated by $A_{0}$ and $u_{1}$ will be of the desired form (as in (2.1)) and will contain $C(\mathscr{P})$ as desired. (This will be shown later.) However, it will not have an approximation to $u$. Now $v_{1} u_{1}=u$, but $v_{1}$ is in $A_{1}$ and not in $A_{0}$. We will apply Berg's technique (see Lemma 1 of Versik [9]) to produce a unitary $z$ in $A_{1}$ such that $z v_{0} z^{*}$ approximates $v_{1}$. We will do this with sufficient care so that $z$ commutes with $u_{1}$ and with $C(\mathscr{P})$. Then the $C^{*}$-algebra generated by $z A_{0} z^{*}$ and $u_{1}$ will have all the desired properties.

Consider the unitary operator $v_{1} v_{0}^{*} \in A_{1}$. From the conditions above, $v_{1} v_{0}^{*}=I$ on $L^{2}(X-\phi(Y))$. Since it is contained in a finite dimensional algebra, its spectrum is finite and we may find $w \in A_{1}$, which is a unitary operator on $L^{2}(\phi(Y))$ such that $w^{N}=v_{1} v_{0}^{*}$ and such that $\|w-I\|<\pi / N \leq \varepsilon$.

Recalling that $u$ carries $L^{2}\left(\phi^{j}(Y)\right)$ isometrically onto $L^{2}\left(\phi^{j+1}(Y)\right)$ for $0 \leq j<N$, define a unitary operator $z \in A_{1}$ by

$$
z= \begin{cases}u^{j} w^{N-j} u^{-j} & \text { on } L^{2}\left(\phi^{j+1}(Y)\right) \quad 0 \leq j<N \\ I & \text { on } L^{2}\left(X-\left(\phi(Y) \cup \cdots \cup \phi^{N}(Y)\right)\right) .\end{cases}
$$

We consider the operator $z v_{0}-v_{1} z$. For $0 \leq j<N$, this operator carries $L^{2}\left(\phi^{j}(Y)\right)$ into $L^{2}\left(\phi^{j+1}(Y)\right)$. First, let us consider the operator on $L^{2}(Y)$, where $z=I$. Now $v_{0}$ carries $L^{2}(Y)$ onto $L^{2}(\phi(Y))$, where $z=w^{N}=v_{1} v_{0}^{*}$, by 2.5. Therefore,

$$
\left(z v_{0}-v_{1} z\right)\left|L^{2}(Y)=\left(v_{1} v_{0}^{*} v_{0}-v_{1}\right)\right| L^{2}(Y)=0 .
$$

Next, let us take $1 \leq j<N$ and consider $z v_{0}-v_{1} z \mid L^{2}\left(\phi^{j}(Y)\right)$. Recall that $z \mid L^{2}\left(\phi^{j}(Y)\right)=u^{j-1} w^{N-j+1} u^{1-j}$. Also note that $v_{0}$ carries $L^{2}\left(\phi^{j}(Y)\right)$ onto $L^{2}\left(\phi^{j+1}(Y)\right)$ where $z=u^{j} w^{N-j} u^{-j}$. Finally, on $L^{2}\left(\phi^{j}(Y)\right)$, we have $v_{0}=u=v_{1}$. Putting this together, we obtain

$$
\begin{aligned}
\left(z v_{0}-v_{1} z\right) \mid L^{2}\left(\phi^{j}(Y)\right) & =u^{j} w^{N-j} u^{-j} u-u u^{j-1} w^{N-j+1} u^{1-j} \\
& =u^{j} w^{N-j}(I-w) u^{1-j}
\end{aligned}
$$

From this we see that

$$
\left\|\left(z v_{0}-v_{1} z\right) \mid L^{2}\left(\phi^{j}(Y)\right)\right\|=\|I-w\|<\pi / N<\varepsilon .
$$


Thirdly, it is immediate from the definitions that on

$$
L^{2}\left(X-\left(Y \cup \phi(Y) \cup \cdots \cup \phi^{N}(Y)\right)\right), v_{0}=u=v_{1} \quad \text { and } z=I, \quad \text { so } z v_{0}-v_{1} z=0 \text {. }
$$

Altogether, we conclude that $\left\|z v_{0}-v_{1} z\right\|<\varepsilon$. Then we have

$$
\left\|z v_{0} z^{*} u_{1}-v_{1} u_{1}\right\|=\left\|z v_{0}-v_{1} z\right\|<\varepsilon .
$$

As we noted earlier, $C(\mathscr{P}) \subset A_{0}$. We now wish to show that $z$ commutes with each element of $C(\mathscr{P})$. This follows immediately from the following two facts. First, $z$ leaves invariant $L^{2}\left(\phi^{j}(Y)\right)$, for each $j=0,1, \ldots, N$, and $z=I$ on $L^{2}\left(X-\left(Y \cup \cdots \cup \phi^{N}(Y)\right)\right)$. Secondly, $Y$ was chosen so that $\phi^{j}(Y)$ is contained in a single element of $\mathscr{P}$, for each $j=0,1, \ldots, N$. (This means that each $f \in C(\mathscr{P})$ acts as a multiple of the identity on each $L^{2}\left(\phi^{j}(Y)\right)$.)

Let $A$ be the $C^{*}$-algebra generated by $z A_{0} z^{*}$ and $u_{1}$. We claim that $A$ satisfies the properties of Proposition 2.1. First, $C(\mathscr{P}) \subset A_{0}$ and $z$ commutes with $C(\mathscr{P})$, so $C(\mathscr{P}) \subset A$. Secondly, $v_{0} \in A_{0}$, so $u^{\prime}=\left(z v_{0} z^{*}\right) u_{1} \in A$ and $\left\|u^{\prime}-u\right\|<\varepsilon$, from (2.6). All that remains to be shown is that

$$
A \simeq\left[M_{J_{1}} \otimes C\left(S^{1}\right)\right] \oplus M_{J_{2}} \oplus \cdots \oplus M_{J_{K}} .
$$

Since $z=I$ on $L^{2}(Y)(2.5)$ and $u_{1}=I$ on $L^{2}(X-Y), z$ and $u_{1}$ commute, so $A$ is unitarily equivalent (via $z$ ) to $B$, the $C^{*}$-algebra generated by $A_{0}$ and $u_{1}$. Let

$$
\hat{u}=\sum_{j=1}^{J_{1}} e_{j J_{1}}^{(1)} u_{1} e_{J_{1} j}^{(1)}+\sum_{k>1} p_{k}
$$

Then $\hat{u}$ is a unitary operator in $B$. It is clear that $\hat{u}$ and $A_{0}$ generate $B$ (as a $C^{*}$-algebra). Moreover, $\hat{u}$ commutes with $A_{0}$ and $\hat{u} p_{k}=p_{k} \hat{u}=p_{k}$ for all $k>1$. All that needs to be shown now is that $\operatorname{sp}(\hat{u})=s p\left(u_{1}\right)=S^{1}$. Recall that $u=v_{1} u_{1}$, so that in $K_{1}\left(C(X) x_{\phi} \mathbb{Z}\right),[u]=\left[v_{1}\right]+\left[u_{1}\right]$. As we noted in $\S 1,[u] \neq 0$, while $\left[v_{1}\right]=0$ since $v_{1} \in A_{1}$, which is a finite dimensional $C^{*}$-algebra. Thus, $\left[u_{1}\right] \neq 0$ and so $s p\left(u_{1}\right)$ must be the entire circle.

This completes the proof of Theorem 2.1.

\section{The general case}

We now examine the general case. We will make the simplifying assumption that $X$ has no non-trivial clopen $\phi$-invariant subsets. (Note that if $Y \subseteq X$ is clopen and $\phi$-invariant, then

$$
\left.C(X) x_{\phi} \mathbb{Z} \cong\left[C(Y) x_{\phi} \mathbb{Z}\right] \oplus\left[C(X-Y) x_{\phi} \mathbb{Z}\right] .\right)
$$

Recall that a $C^{*}$-algebra $A$ is said to have the cancellation property if, whenever $U, V$ and $W$ are finitely generated projective left $A$-modules such that $U \oplus W \cong V \oplus$ $W$, then we have $U \cong V$. In terms of projections, this says that whenever projections $p$ and $q$ in $A$ (or $M_{n} \otimes A$ ) determine the same element in $K_{0}(A)$, then they are actually unitarily equivalent. We refer the reader to Rieffel [8] for a more complete treatment.

THEOREM 3.1. Suppose that $X$ has no non-trivial, clopen, $\phi$-invariant subsets. The following are equivalent. 
(i) The invertible elements in $C(X) x_{\phi} \mathbb{Z}$ are dense.

(ii) The $C^{*}$-algebra $C(X) x_{\phi} \mathbb{Z}$ has the cancellation property.

(iii) There is a unique minimal set for $\phi$.

Remark. Regarding condition (iii), a standard Zorn's Lemma argument shows that there is always at least one minimal set.

Proof. The implication (i) $\Rightarrow$ (ii) is valid for all $C^{*}$-algebras. (See Blackadar [1].)

Let us now show that (iii) $\Rightarrow$ (i). Let us denote the unique minimal set by $Y$. Consider the following short exact sequence (see Zeller-Meier [12]).

$$
0 \rightarrow C_{0}(X-Y) x_{\phi} \mathbb{Z} \stackrel{i}{\rightarrow} C(X) x_{\phi} \mathbb{Z} \stackrel{q}{\rightarrow} C(Y) x_{\phi} \mathbb{Z} \rightarrow 0 .
$$

For brevity, we shall denote these $C^{*}$-algebras by $I, A$ and $B$, respectively.

If $x$ is any point of $X$, the set of accumulation points of $\left\{\phi^{n}(x) \mid n \in \mathbb{Z}\right\}$ is closed and $\phi$-invariant and therefore must contain $Y$. We conclude that $Y$ satisfies the condition that $\bigcup_{n \in \mathbb{Z}} \phi^{n}(W)=X$, whenever $W$ is a clopen set containing $Y$. So by Theorem 2.2 of Poon [7], $I$ is an $A F$-algebra. We conclude that the invertible elements in the unitalized $C^{*}$-algebra $I^{\sim}$ are dense. From Corollary 2.3, we also know that the invertible elements of $B$ are dense. From Corollary 2.4, we see that every invertible in $B$ has the form $u_{Y, \phi}^{k} v$, where $k \in \mathbb{Z}$ and $v \in G L_{1}^{0}(B)$, Clearly, $u_{Y, \phi}$ lifts to $u_{X, \phi} \in A$ and it is well-known that every element of $G L_{1}^{0}(B)$ may be lifted to something in $G L_{1}^{0}(A)$ (3.4.4 of Blackadar [1]). We conclude that every invertible in $B$ may be lifted to an invertible in $A$.

We are now ready to show that the invertibles in $A$ are dense. Let $a \in A$ and $\varepsilon>0$. There is an invertible $b \in B$ such that $\|q(a)-b\|<\varepsilon / 3$ and there is an invertible $c \in A$ such that $q(c)=b$. Since $\|q(a)-q(c)\|<\varepsilon / 3$, we may find $d \in I$ such that $\|a-c-d\|<2 \varepsilon / 3$. We may find an invertible $e \in I^{-}$such that $\left\|\left(1+c^{-1} d\right)-e\right\|<$ $\varepsilon / 3\|c\|$. Then $c e$ is invertible and

$$
\|a-c e\| \leq\left\|a-c\left(1+c^{-1} d\right)\right\|+\|c\|\left\|\left(1+c^{-1} d\right)-e\right\|<2 \varepsilon / 3+\varepsilon / 3=\varepsilon .
$$

Finally, we consider (ii) $\Rightarrow$ (iii). Suppose that (iii) is false. That is, there are (at least) two distinct minimal sets, $Y_{1}$ and $Y_{2}$, for $\phi$. Since these are distinct, $Y_{1} \cap Y_{2}$ will be a proper subset of either $Y_{1}$ or $Y_{2}$. Moreover, it is closed and $\phi$-invariant so, by the minimality of $Y_{1}$ and $Y_{2}$, must be empty. We consider the short exact sequence

$$
0 \rightarrow C_{0}\left(X-Y_{1}-Y_{2}\right) x_{\phi} \mathbb{Z} \stackrel{i}{\rightarrow} C(X) x_{\phi} \mathbb{Z} \stackrel{q}{\rightarrow} C\left(Y_{1} \cup Y_{2}\right) x_{\phi} \mathbb{Z} \rightarrow 0
$$

Again, we denote these $C^{*}$-algebras by $I, A$ and $B$, respectively, and note that $C\left(Y_{1} \cup Y_{2}\right) x_{\phi} \mathbb{Z}$ is *-isomorphic to $\left[C\left(Y_{1}\right) x_{\phi} \mathbb{Z}\right] \oplus\left[C\left(Y_{2}\right) x_{\phi} \mathbb{Z}\right]$. Thus, $K_{1}(B) \cong \mathbb{Z} \oplus \mathbb{Z}$, and is generated by the classes of

$$
u_{1}=u_{Y_{1} \cup Y_{2}, \phi} X_{Y_{1}}+\chi_{Y_{2}} \text { and } u_{2}=u_{Y_{1} \cup Y_{2}, \phi} \chi_{Y_{2}}+\chi_{Y_{1}} \text {. }
$$

It is clear that $q_{*}: K_{1}(A) \rightarrow K_{1}(B)$ takes $\left[u_{X, \phi}\right]$ to $\left[u_{Y_{1} \cup Y_{2}, \phi}\right]=\left[u_{1} u_{2}\right.$ ]. Recall that $\left[u_{X, \phi}\right]$ generates $K_{1}(A)$ so we see that $\left[u_{1}\right]$ is not in the image of $q_{*}$ and so $\partial\left[u_{1}\right] \neq 0$, where $\partial$ denotes the index map $K_{1}(B) \rightarrow K_{0}(I)$. Let us compute $\partial\left[u_{1}\right]$. Let $E$ be any clopen set in $X$ containing $Y_{1}$ and disjoint from $Y_{2}$. Let $F=X-(E \cup \phi(E))$. Note that since $\phi\left(Y_{2}\right)=Y_{2}, \phi(E)$ is also disjoint from $Y_{2}$ so $Y_{2} \subseteq F$. Let $a=u_{X, \phi} \chi_{E}+\chi_{F}$. 
Then $a$ is a partial isometry in $A$ with $a^{*} a=\chi_{E \cup F}$ and $a a^{*}=\chi_{\phi(E) \cup F}$. Also note that $q(a)=u_{1}$. So, $\partial\left[u_{1}\right]=\left[\chi_{\phi(E)-E}\right]-\left[\chi_{E-\phi(E)}\right]$. Denote the projections by $p_{1}$ and $p_{2}$, respectively. Note that they are both elements of $I$. As we have already observed $\left[p_{1}\right]-\left[p_{2}\right]=\partial[v] \neq 0$ in $K_{0}(I)$. However, in $K_{0}(A),\left[i\left(p_{i}\right)\right]-\left[i\left(p_{2}\right)\right]=i_{*} \partial\left[u_{1}\right]=0$. Suppose that there is a unitary $w$ in $A$ such that $w p_{1} w^{*}=p_{2}$. Then $w p_{1}$ is a partial isometry in $I$ (since $\left.p_{1} \in I\right)$ with $\left(w p_{1}\right)\left(w p_{1}\right)^{*}=p_{2}$ and $\left(w p_{1}\right)^{*}\left(w p_{1}\right)=p_{1}$. This would imply $\left[p_{2}\right]=\left[p_{1}\right]$ in $K_{0}(I)$. We conclude that while $p_{1}$ and $p_{2}$ determine the same class in $K_{0}(A)$, they are not unitarily equivalent. Therefore, $A$ fails to have the cancellation property.

\section{Residual finiteness}

In this section, we examine the relationship between the results of $\S 3$ and the property of residual finiteness of the $C^{*}$-algebra $C(X) x_{\phi} \mathbb{Z}$. We take a moment to discuss, in general terms, why this is relevant. There are two canonical types of examples of unital $C^{*}$-algebras in which the invertibles are not dense: $C(M)$, where $M$ is a compact metrizable space whose dimension is at least two (see Rieffel [8]) and any $C^{*}$-algebra containing a non-unitary isometry. Recall that a $C^{*}$ algebra, $A$, is called finite if every isometry in $A$ is actually a unitary. Let us take this second phenomenon one step further. A unital $C^{*}$-algebra, $A$, is called residually finite if every quotient of $A$ is finite. If $A$ has a quotient, $A / I$, which is not finite, then the invertibles in $A / I$ are not dense and it follows that the invertibles in $A$ are not dense. We summarize by saying that there are two obstructions to the invertibles in a $C^{*}$-algebra being dense: a topological one and finiteness one.

In our situation, $X$ is zero-dimensional and the group $\mathbb{Z}$ has a one-dimensional dual. The $C^{*}$-algebra $C(X) x_{\phi} \mathbb{Z}$ is a 'non-commutative $X \times \hat{\mathbb{Z}} \cong X \times S^{1}$, which is one-dimensional. Thus, one would not expect 'topological' obstructions to the invertibles being dense, only finiteness. However, we shall produce an example of a $C(X) x_{\phi} \mathbb{Z}$ which is residually finite and yet the invertibles are not dense.

We begin with a necessary and sufficient dynamical condition for $C(X) x_{\phi} \mathbb{Z}$ to be residually finite. This is a more or less straightforward consequence of a result of M. Pimsner.

We begin by making some definitions. Fix a metric $d$ on $X$. For a positive real number $\varepsilon$, and points $x$ and $y$ in $X$, an $\varepsilon$-chain from $x$ to $y$ is a finite sequence of points $\left(x_{1}, x_{2}, \ldots, x_{n}\right)$ in $X$ such that $x_{1}=x, x_{n}=y$ and $d\left(\phi\left(x_{i}\right), x_{i+1}\right)<\varepsilon$, for $i=1$, $2, \ldots, n-1$. A point $x$ in $X$ is chain recurrent for $\phi$ if, for every $\varepsilon>0$, there is an $\varepsilon$-chain from $x$ to $x$ (of length at least two).

We remark that a point of $x$ may be chain recurrent for $\phi$ in $X$, but not when considered as a point in some $\phi$-invariant closed subset of $X$, since the $\varepsilon$-chains may not lie in the subset.

For any $x$ in $X$, we let $\omega^{+}(x)$ and $\omega^{-}(x)$ denote the accumulation points of $\left\{\phi^{n}(x) \mid n \geq 0\right\}$ and $\left\{\phi^{n}(x) \mid n \leq 0\right\}$, respectively. These are both closed and $\phi$-invariant.

ThEOREM 4.1. (Pimsner [5].) The $C^{*}$-algebra $C(X) x_{\phi} \mathbb{Z}$ is finite if and only if each point of $X$ is chain recurrent for $\phi$. 
Pimsner actually showed much more; namely that these conditions are equivalent to $C(X) x_{\phi} \mathbb{Z}$ being $A F$-embeddable.

THEOREM 4.2. The following are equivalent.

(i) $C(X) x_{\phi} \mathbb{Z}$ is residually finite.

(ii) For every $x$ in $X$ and every $\varepsilon>0$, there is an $\varepsilon$-chain, $\left(x_{1}, x_{2}, \ldots, x_{n}\right)$, from $x$ to $x$, with each $x_{i} \in\left\{\phi^{n}(x) \mid n \in \mathbb{Z}\right\}$.

(iii) For each $x$ in $X, \omega^{+}(x) \cap \omega^{-}(x)$ is not empty.

Proof. (i) $\Rightarrow$ (iii). We prove the contrapositive. Suppose there is an $x$ in $X$ with $\omega^{+}(x) \cap \omega^{-}(x)$ empty. Let $Y=\left\{\phi^{n}(x) \mid n \in \mathbb{Z}\right\} \cup \omega^{+}(x) \cup \omega^{-}(x)$ and let $E=$ $\left\{\phi^{n}(x) \mid n \geq 0\right\} \cup \omega^{+}(x)$. Then $C(Y) x_{\phi} \mathbb{Z}$ is a quotient of $C(X) x_{\phi} \mathbb{Z}$ which contains $u_{Y, \phi} \chi_{E}+\chi_{Y-E}$ which is a non-unitary isometry. The proof of (iii) $\Rightarrow$ (ii) is straightforward, so we omit it.

(ii) $\Rightarrow(i)$. We need to show that for any ideal $I$ in $C(X) x_{\phi} \mathbb{Z}$, the quotient $C(X) x_{\phi} \mathbb{Z} / I$ is finite. We begin with the following special case. Suppose $Y$ a closed $\phi$-invariant subset of $X$. We consider $I=C_{0}(X-Y) x_{\phi} \mathbb{Z}$. In this case, $C(X) x_{\phi} \mathbb{Z} / I \cong$ $C(Y) x_{\phi} \mathbb{Z}$ and we will apply 4.1 . Let $x$ be any point in $Y$. Since $Y$ is $\phi$-invariant, the entire orbit of $x$ under $\phi$ is in $Y$. Therefore, by (ii), we can find, for any $\varepsilon>0$, an $\varepsilon$-chain from $x$ to $x$ within $Y$. By $4.1, C(Y) x_{\phi} \mathbb{Z}$ is finite.

As for the general ideal $I$, let us suppose that the quotient $C(X) x_{\phi} \mathbb{Z} / I$, which we denote $B$, is not finite. Then we may find an irreducible representation $\pi$ of $B$ (and also of $C(X) x_{\phi} \mathbb{Z}$ ) on the Hilbert space $\mathscr{H}$ such that $\pi(B)$ is not finite. By analyzing $\pi \mid C(X)$, it can be shown that $\mathscr{H}$ is isomorphic to $L^{2}(X, \mu)$, for some (possibly infinite) $\phi$-invariant measure $\mu$ on $X$ and $C(X)$ is represented on $L^{2}(X, \mu)$ as multiplication operators. Since $\pi(B)$ is not finite, $\mathscr{H}$ cannot be finite-dimensional. Thus, $Y=$ support $(\mu)$ is closed, $\phi$-invariant and infinite. Since $\pi$ is irreducible, $Y$ must contain a point whose orbit under $\phi$ is infinite. We conclude that $\pi(B)$ is actually *-isomorphic to $C(Y) x_{\phi} \mathbb{Z}$, which we have already seen is finite. This contradiction establishes the result.

We conclude by giving an example of $X$ and $\phi$ such that $C(X) x_{\phi} \mathbb{Z}$ is residually finite but the invertibles are not dense.

We provide a picture of $X$ in figure 1. Each box is a clopen set. The points $x_{0}$ and $x_{1}$ are fixed by $\phi$. Also, $\phi$ is such that $\phi^{-1}\left(E_{1}\right) \subseteq E, \phi\left(E_{1}\right)=E_{2}, \phi\left(E_{2}\right) \subseteq F$,

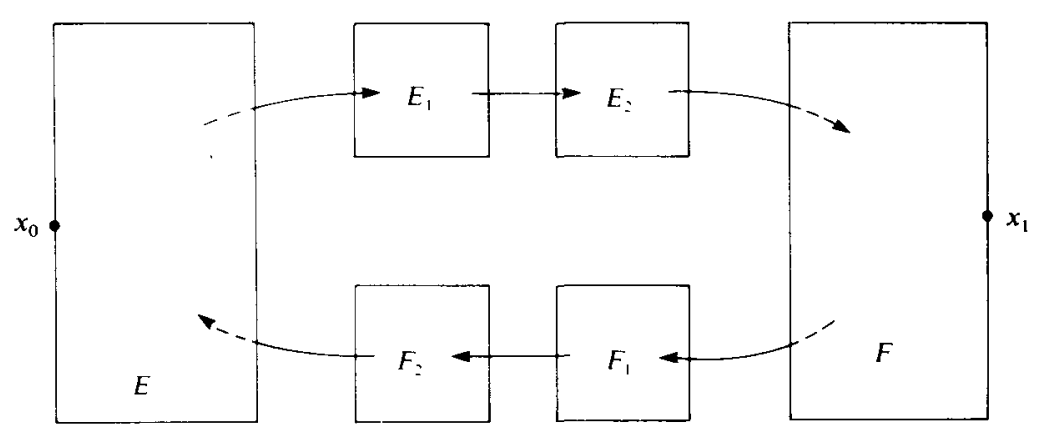

FIGURE 1 


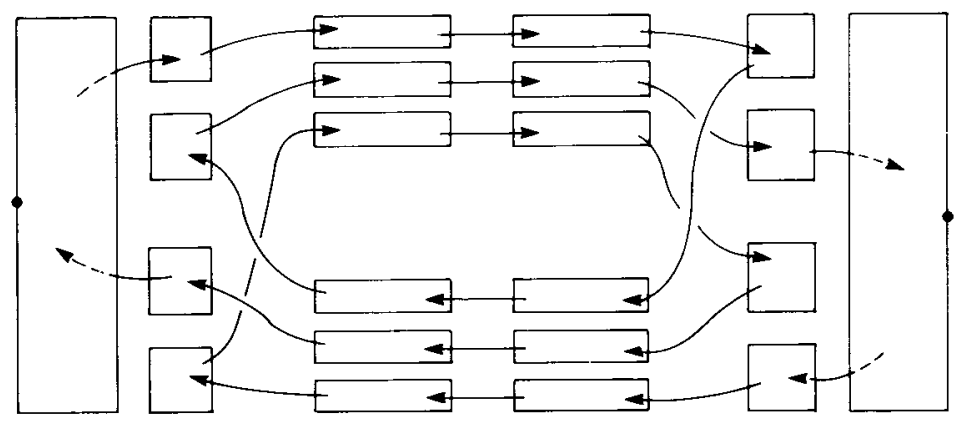

FIGURE 2

$\phi^{-1}\left(F_{1}\right) \subseteq F, \phi\left(F_{1}\right)=F_{2}$ and $\phi\left(F_{2}\right) \subseteq E$. We also show a 'finer' picture of $X$ in figure 2 , with arrows indicating $\phi$.

From these figures, one can continue inductively to give a rigorous definition of $\phi$ and $X$. It is clear that there are no clopen $\phi$-invariant subsets of $X$, while $\left\{x_{0}\right\}$ and $\left\{x_{1}\right\}$ are each minimal sets for $\phi$. Thus, the invertibles in $C(X) x_{\phi} \mathbb{Z}$ are not dense. It can also be seen that, for any $x$ in $X, \omega^{+}(x) \cap \omega^{-}(x)$ contains either $x_{0}$ or $x_{1}$ (or both) and so $C(X) x_{\phi} \mathbb{Z}$ is residually finite.

\section{REFERENCES}

[1] B. Blackadar. K-theory for operator algebras. Math. Sci. Research Institute Publications 5, SpringerVerlag: Berlin-Heidelberg-New York, 1986.

[2] I. P. Cornfeld, S. V. Fomin \& Ya. G. Sinai. Ergodic theory. Grundlehren der math. Wiss. 245, Springer-Verlag: Berlin-Heidelberg-New York, 1982.

[3] E. G. Effros. Dimensions and $C^{*}$-algebras. Conference Board Math. Sci. 46, American Mathematical Society: Providence, RI, 1981.

[4] G. K. Pedersen. $C^{*}$-algebras and their automorphism groups. London Mathematical Society Monographs 14, Academic Press: London, 1979.

[5] M. V. Pimsner. Embedding some transformation group $C^{*}$-algebras into AF-algebras. Ergod. Th. \& Dynam. Sys. 3 (1983), 613-626.

[6] M. Pimsner \& D. Voiculescu. Exact sequences for $K$-groups and ext-groups of certain cross-product $C^{*}$-algebras. J. Operator Theory 4 (1980), 93-118.

[7] Y. T. Poon. AF-subalgebras of certain crossed products, preprint.

[8] M. A. Rieffel. Dimensions and stable rank in the $K$-theory of $C^{*}$-algebras. Proc. London Math. Soc. (3), 46 (1983), 301-333.

[9] A. M. Versik. Uniform algebraic approximation of shift and multiplication operators. Sov. Math. Dokl. 24 ( 1981 ) 97-100.

[10] A. M. Versik. A theorem on periodical Markov approximation in ergodic theory. Ergodic Theory and Related Topics (Vitte, 1981), 195-206, Math. Res. 12, Akademie-Verlag: Berlin, 1981.

[11] G. W. Whitehead. Elements of homotopy theory. Graduate Texts in Mathematics 61, Springer-Verlag: Berlin-Heidelberg-New York, 1978.

[12] G. Zeller-Meier. Produits croises d'une $C^{*}$-algebre par un group d'automorphismes. J. Math. Pures et Appl. 47 (1968), 101-239. 